\title{
Estudio coproparasitológico de helmintos gastrointestinales en zopilotes negros (Coragyps atratus) de Livingston, Izabal, Guatemala
}

\author{
A coproparasitological survey of gastrointestinal helminths in black vultures \\ (Coragyps atratus) from Livingston, Izabal, Guatemala
}

\section{Estudo coproparasitológico de helmintos gastrointestinais em urubus-pretos (Coragyps atratus) de Livingston, Izabal, Guatemala}

\section{Jessica Callejas-Martínez ${ }^{1}$}

1 Universidad de San Carlos de Guatemala, Facultad de Medicina Veterinaria y Zootecnia, Escuela de Medicina Veterinaria, Ciudad de Guatemala, Guatemala. E-mail: jesscm1436@gmail.com

Recibido: 2 de abril de 2019 Corregido: 16 de agosto de 2019 Aceptado: 12 de setiembre de 2019

Resumen: El objetivo del estudio fue determinar la presencia de helmintos gastrointestinales, a través del análisis coproparasitológico, en zopilotes negros (Coragyps atratus) de un área de Livingston, Izabal, Guatemala. Se analizó un total de 32 muestras fecales de estas aves por medio del método directo y el de flotación, con una solución sobresaturada de azúcar. De las 32 muestras analizadas, 21 (65.6\%) resultaron positivas a la presencia de huevos de helmintos gastrointestinales; de estas, 20 (95.2\%) presentaron huevos de trematodos; $3(14.3 \%)$ presentaron huevos de nematodos; en $1(4.8 \%)$ se encontró huevos de un acantocéfalo. Los huevos de un trematodo, de la superfamilia Diplostomoidea, fueron los más comúnmente observados, detectándose en $20(62.5 \%)$ de las 32 muestras analizadas. Además, se encontraron huevos de un trematodo de la familia Echinostomatidae; de nematodos del género Heterakis y de las familias Ascarididae y Trichostrongylidae, así como huevos de Acanthocephala. De acuerdo con lo detectado a través del análisis coproparasitológico, los zopilotes negros evaluados presentaron helmintos gastrointestinales, predominando una especie de trematodo de la superfamilia Diplostomoidea. Los hallazgos de esta investigación constituyen un primer acercamiento al conocimiento de la fauna helmintológica gastrointestinal de Coragyps atratus en Guatemala.

Palabras clave: Coragyps atratus, helmintos, huevos de helmintos, trematodos.

\begin{abstract}
The aim of this study was to determine the presence of gastrointestinal helminths in black vultures (Coragyps atratus) from an area in Livingston, Izabal, Guatemala, using coproparasitological analysis. Thirty-two fecal samples from these birds were analyzed by the direct smear method and the saturated sugar solution flotation method. Out of the 32 analyzed fecal samples, 21 (65.6\%) tested positive for gastrointestinal helminth ova and, out of these, 20 (95.2\%) presented trematode ova, 3 (14.3\%) nematode ova, and $1(4.8 \%)$ acanthocephalan ova. Eggs of a trematode of the superfamily Diplostomoidea were the most commonly observed, being detected in $20(62.5 \%)$ out of the 32 samples analyzed. Furthermore, samples contained trematode eggs from the family Echinostomatidae, nematode eggs from the genus Heterakis and the families Ascarididae and Trichostrongylidae, as well as eggs of Acanthocephala. Based on the coproparasitological analysis, the evaluated black vultures had gastrointestinal helminths, with the predominance of a trematode species of the superfamily Diplostomoidea. The findings of this research constitute the first approach to learning about the gastrointestinal helminthological fauna of Coragyps atratus in Guatemala.
\end{abstract}

Keywords: Coragyps atratus, helminths, helminth ova, trematodes. 
Rev. Ciencias Veterinarias, Vol. 37, N² 2, [32-43], E-ISSN: 2215-4507, julio-diciembre, 2019

DOI: https://doi.org/10.15359/rcv.37-2.4

URL: http://www.revistas.una.ac.cr/index.php/veterinaria/index

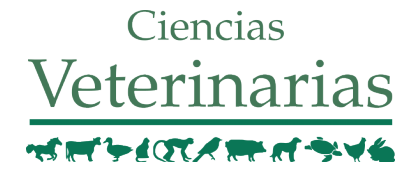

\begin{abstract}
Resumo: O objetivo deste estudo foi determinar a presença de helmintos gastrointestinais, através de análises coproparasitológicas, em urubus pretos (Coragyps atratus) de uma área de Livingston, Izabal, Guatemala. Um total de 32 amostras fecais dessas aves foram analisadas pelo método direto e pelo método de flotação com solução de açúcar supersaturada. Das 32 amostras analisadas, 21 (65,6\%) foram positivas para a presença de ovos de helmintos gastrointestinais e, dessas, 20 (95,2\%) apresentaram ovos de trematódeos, 3 $(14,3 \%)$ apresentaram ovos de nematóides e $1(4,8 \%)$ apresentou ovos de um acantocéfalo. Os ovos de um trematódeo da superfamília Diplostomoidea foram os mais observados, sendo detectados em 20 (62,5\%) das 32 amostras analisadas. Além disso, foram encontrados ovos de um trematódeo da família Echinostomatidae, de nematóides do gênero Heterakis e das famílias Ascarididae e Trichostrongylidae, além de ovos de Acanthocephala. De acordo com os resultados da análise coproparasitológica, os urubus pretos avaliados apresentaram helmintos gastrointestinais, com predomínio de uma espécie de trematódeo da superfamília Diplostomoidea. Os resultados desta pesquisa constituem uma primeira abordagem ao conhecimento da fauna helmintológica gastrointestinal de Coragyps atratus na Guatemala.
\end{abstract}

Palavras-chave: Coragyps atratus, helmintos, ovos de helmintos, trematódeos

\title{
Introducción
}

El zopilote negro (Coragyps atratus) posee un amplio rango de distribución. Abarca parte de Norteamérica, toda Centroamérica, la mayor parte de Sudamérica y algunas islas del Caribe (Raffaele et al. 2003; Houston \& Kirwan 2019). Habita, principalmente, áreas abiertas o semiabiertas, zonas con poblaciones humanas y otras cercanas a cuerpos de agua. Es usual ver grupos numerosos de estas aves en basureros, mataderos o sitios donde tienen acceso a desechos que sirven de alimento (Ferguson-Lees \& Christie 2001; Avery \& Lowney 2016). Su dieta es muy variada: incluye carroña (de cualquier animal doméstico o silvestre); animales vivos pequeños, como crías de aves, reptiles, peces y artrópodos, incluso crías recién nacidas de algunos mamíferos; además de huevos, frutas, heces de animales y restos de basura (Ferguson-Lees \& Christie 2001; Ballejo \& De Santis 2013; Avery \& Lowney 2016).

A pesar de que el zopilote negro es un ave común, e incluso abundante en muchos lugares del continente americano (Bildstein et al. 2007; Houston \& Kirwan 2019), existe poca información sobre su fauna helmintológica. En el cuadro 1 se muestran los helmintos gastrointestinales que se han encontrado en estas aves.

Debido a que los zopilotes negros son aves asociadas a actividades humanas como: la ganadería, la pesca, la agricultura o la concentración de desechos en diversos sitios, podrían actuar como diseminadores de agentes patógenos como helmintos y otros parásitos que afectan a los seres humanos y a animales domésticos o silvestres (Schlatter et al. 1978; Avery \& Lowney 2016). Además, aunque la presencia de parásitos en animales silvestres de vida libre, rara vez se asocia con enfermedad, pues ambos, tanto parásitos como hospederos, tienden a coexistir en un estado de equilibrio, se pueden producir efectos adversos en un animal parasitado cuando se asocian otros factores como: malnutrición, inmunidad reducida, enfermedades concomitantes, alta carga parasitaria, contaminación ambiental, pérdida del hábitat, entre otros (Borgsteede 1996; Krone 2007; Goater et al. 2014). Los parásitos pueden tener un impacto en los individuos y en las poblaciones de animales silvestres y, por consiguiente, en la salud de los ecosistemas, por lo cual, el estudio de estos agentes permite comprender mejor los efectos que producen (Borgsteede 1996; Loker \& Hofkin 2015). Por consiguiente, se hace necesario aumentar el conocimiento en el campo de la parasitología en zopilotes negros. 
Cuadro 1. Registros de helmintos gastrointestinales en Coragyps atratus

\begin{tabular}{|c|c|c|c|}
\hline Helmintos & Localidad & Ubicación en el hospedero & Referencia \\
\hline \multicolumn{4}{|l|}{ Trematodos } \\
\hline \multirow{5}{*}{ Paryphostomum segregatum } & Brasil & $\mathrm{ND}^{*}$ & Noronha et al. 2009 \\
\hline & Brasil & Detección de huevos en heces & Lie \& Basch 1967 \\
\hline & Argentina & Intestino & Drago \& Lunaschi 2011 \\
\hline & Paraguay & ND & Kostadinova et al. 2002 \\
\hline & Venezuela & ND & Alves-Pinto 2013 \\
\hline \multirow[t]{3}{*}{ Strigea vaginata } & Brasil & Intestino, músculo & Noronha et al. 2009 \\
\hline & Argentina & Intestino & Drago \& Lunaschi 2011 \\
\hline & Venezuela & ND & Lunaschi \& Drago 2009 \\
\hline Strigea falconis brasiliana & Brasil & Intestino & Noronha et al. 2009 \\
\hline Strigea sp. & Brasil & Intestino & Noronha et al. 2009 \\
\hline Strigeidae & Brasil & Detección de huevos en heces & Lie \& Basch 1967 \\
\hline $\begin{array}{c}\text { Apatemon (Australapatemon) } \\
\text { bdellocystis }\end{array}$ & Brasil & Intestino & Noronha et al. 2009 \\
\hline Ascocotyle sp. & Brasil & Intestino & Noronha et al. 2009 \\
\hline Petasiger sp. & Argentina & Intestino & Drago \& Lunaschi 2011 \\
\hline \multicolumn{4}{|l|}{ Nematodos } \\
\hline Tetrameres paradoxus & Brasil & Proventrículo & Vicente et al. 1995 \\
\hline Tetrameres sp. & Brasil & Proventrículo & Vicente et al. 1995 \\
\hline Heterakis sp. & Brasil & Detección de huevos en heces & Frasson et al. 2016 \\
\hline \multicolumn{4}{|l|}{ Acantocéfalos } \\
\hline Oligacanthorhynchus spira & Brasil & Intestino & Travassos 1917 \\
\hline
\end{tabular}

ND: No definida

La información existente sobre los helmintos gastrointestinales que parasitan a estas aves corresponde a hallazgos en países sudamericanos (Brasil, Argentina, Paraguay y Venezuela). No se tiene conocimiento de que existan reportes o estudios al respecto en Guatemala. Además, sólo tres de los hallazgos mencionados fueron obtenidos por medio del análisis fecal en aves vivas (Lie \& Basch 1967; Frasson et al. 2016); el resto corresponden a hallazgos post-mortem. Por lo tanto, el objetivo del presente estudio fue determinar la presencia de helmintos gastrointestinales a través del análisis coproparasitológico en zopilotes negros de un área de Livingston, Izabal, Guatemala.

\section{Materiales y métodos}

El estudio se realizó en la Fundación Protectora de Animales en vías de Extinción (FAE) ubicada en las coordenadas $15^{\circ} 35^{\prime} 07.8^{\prime \prime} \mathrm{N}, 88^{\circ} 56^{\prime} 39.0^{\prime \prime O}$ a una altura aproximada de $10 \mathrm{msnm}$ en el municipio de Livingston, departamento de Izabal, Guatemala, en un área de 50.02 ha. La zona de vida en la región 
Rev. Ciencias Veterinarias, Vol. 37, N² 2, [32-43], E-ISSN: 2215-4507, julio-diciembre, 2019

DOI: https://doi.org/10.15359/rcv.37-2.4

Ciencias

URL: http://www.revistas.una.ac.cr/index.php/veterinaria/index

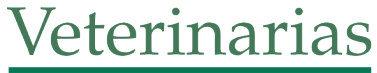

es el bosque muy húmedo tropical según el sistema de clasificación de Holdridge (De la Cruz 1982). El municipio de Livingston presenta una temperatura media anual de $26^{\circ} \mathrm{C}$ y una humedad relativa media del 82\% (INSIVUMEH 2018).

Durante los meses de julio y agosto de 2018 se recolectaron 32 muestras fecales de zopilotes negros de vida libre que frecuentaban el área. Para ello se identificaron posaderos o sitios en los cuales se congregaban varios individuos, lo cual ocurría principalmente en las cercanías de algunos recintos de animales que se encuentran dentro de la FAE. Se recolectaron entre 1-4 muestras por día, en días no consecutivos, en tres de estos sitios; el primero se ubica a una distancia lineal aproximada de $400 \mathrm{~m}$ del segundo y este a $300 \mathrm{~m}$ del tercero.

Las heces con apariencia fresca se recogieron del suelo utilizando guantes de látex y fueron transportadas dentro de estos, en bolsas Ziploc ${ }^{\circledast}$, al laboratorio de la clínica veterinaria de la FAE para realizar una evaluación coproparasitológica. Cuando no fue posible realizar esta evaluación inmediatamente después de la recolección de las muestras, estas se mantuvieron en refrigeración hasta procesarlas en el laboratorio el mismo día de la toma.

El análisis de las muestras fecales se realizó a través del método directo y del método de flotación con una solución sobresaturada de azúcar (Figueroa-Hernández \& Rodríguez-Zea 2007; Zajac \& Conboy 2012). Se procedió a realizar la identificación de los huevos encontrados con base en sus características morfológicas, realizando la comparación con registros fotográficos incluidos en publicaciones de diversos autores (Basch et al. 1973; Hong et al. 1984; Chai et al. 1990; Thienpont et al. 2003; Krone 2007; Zajac \& Conboy 2012; Bowman 2014; Toledo et al. 2014; Taylor et al. 2016; Globokar et al. 2017).

\section{Resultados}

Se analizaron 32 muestras fecales de zopilotes negros de las cuales $21(65.6 \%)$ resultaron positivas a la presencia de huevos de helmintos gastrointestinales. Se detectaron huevos de trematodos en 20 (95.2\%) de las 21 muestras positivas, huevos de nematodos en 3 (14.3\%) y huevos de un acantocéfalo en 1 (4.8\%). No se detectaron huevos o proglótidos de cestodos.

Los huevos encontrados se identificaron como pertenecientes a trematodos de la superfamilia Diplostomoidea y de la familia Echinostomatidae, a nematodos del género Heterakis y de las familias Ascarididae y Trichostrongylidae y a un acantocéfalo de una especie no identificada. Los huevos de Diplostomoidea fueron los más comúnmente observados, detectándose en 20 (62.5\%) de las 32 muestras analizadas (Cuadro 2, Figura 1).

En 5 (23.8\%) de las 21 muestras positivas se encontraron huevos de más de un taxón de helminto, de la siguiente forma: huevos de Diplostomoidea + Echinostomatidae en 2 muestras, de Diplostomoidea + Ascarididae en 1 muestra, de Diplostomoidea + Heterakis sp. en 1 muestra y de Acanthocephala + Trichostrongylidae en 1 muestra. En ninguna muestra se observaron huevos de más de dos taxones.

De las 21 muestras positivas 21 (100\%) fueron detectadas por el método directo y 3 (14.3\%) por el método de flotación con una solución sobresaturada de azúcar. Como se observa en el cuadro 3 todos los huevos de trematodos y los de acantocéfalo se detectaron con el método directo y los huevos de nematodos se detectaron principalmente con el método de flotación. 


\section{Ciencias}

Cuadro 2. Número de muestras fecales de Coragyps atratus positivas a la presencia de huevos de helmintos gastrointestinales según taxón

\begin{tabular}{lcc}
\hline \multicolumn{1}{c}{ Taxón } & No. de muestras positivas & $\%^{*}$ \\
\hline Trematodos & 20 & 62.5 \\
Diplostomoidea & 2 & 6.3 \\
Echinostomatidae & 1 & \\
Nematodos & 1 & 3.1 \\
Heterakis sp. & 1 & 3.1 \\
Ascarididae & & 3.1 \\
Trichostrongylidae & 1 & \\
Acantocéfalos & & 3.1 \\
Acanthocephala &
\end{tabular}

${ }^{*}$ Calculado a partir del total de muestras analizadas (32)
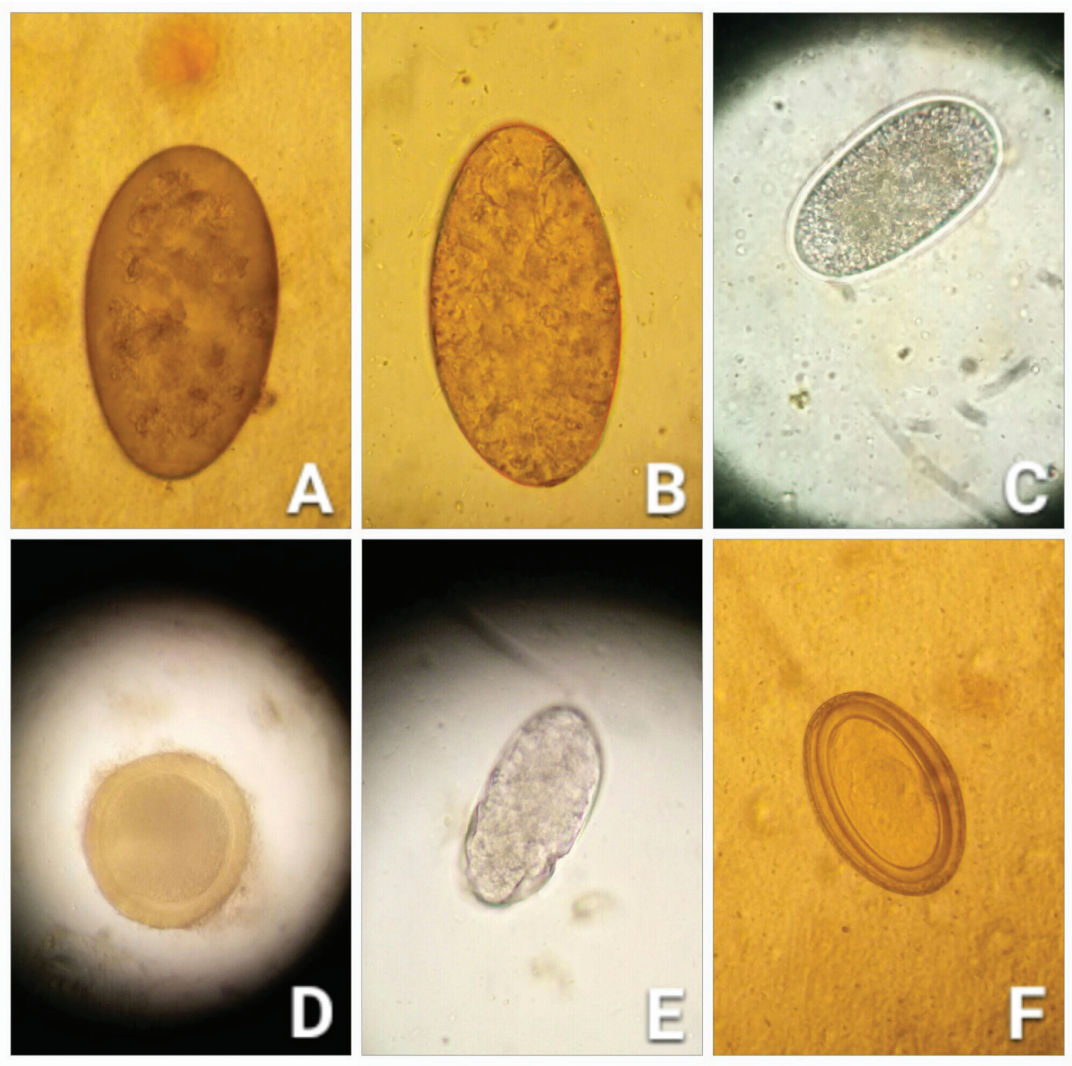

Figura 1. Huevos de helmintos gastrointestinales observados en muestras fecales de Coragyps atratus. (A) Diplostomoidea, 40x, método directo; (B) Echinostomatidae, 40x, método directo; (C) Heterakis sp., 40x, flotación; (D) Ascarididae (probablemente Porrocaecum sp.), 40x, método directo; (E) Trichostrongylidae, 40x, flotación; (F) Acanthocephala, 40x, método directo. 
Rev. Ciencias Veterinarias, Vol. 37, N² 2, [32-43], E-ISSN: 2215-4507, julio-diciembre, 2019

DOI: https://doi.org/10.15359/rcv.37-2.4

URL: http://www.revistas.una.ac.cr/index.php/veterinaria/index

Cuadro 3. Número de muestras fecales de Coragyps atratus positivas a la presencia de huevos de helmintos gastrointestinales según método diagnóstico.

\begin{tabular}{lccc}
\hline \multicolumn{1}{c}{ Taxón } & $\begin{array}{c}\text { Total de muestras } \\
\text { positivas }\end{array}$ & $\begin{array}{c}\text { Muestras positivas con el } \\
\text { método directo }\end{array}$ & $\begin{array}{c}\text { Muestras positivas con el } \\
\text { método de flotación }\end{array}$ \\
\hline $\begin{array}{l}\text { Trematodos } \\
\text { Diplostomoidea }\end{array}$ & 20 & $20(100)$ & No.(\%) \\
$\quad \begin{array}{l}\text { Echinostomatidae } \\
\text { Nematodos }\end{array}$ & 2 & $2(100)$ & 0 \\
$\quad$ Heterakis sp. & 1 & 0 & 0 \\
Ascarididae & 1 & $1(100)$ & $1(100)$ \\
Trichostrongylidae & 1 & 0 & $1(100)$ \\
Acantocéfalos & & & $1(100)$ \\
Acanthocephala & 1 & $1(100)$ & 0 \\
\hline
\end{tabular}

\section{Discusión}

El porcentaje de positividad (65.6\%) a la presencia de huevos de helmintos gastrointestinales en Coragyps atratus obtenido en el presente estudio difiere con lo reportado por Andery et al. (2013), quienes no encontraron helmintos gastrointestinales en ninguno de los cadáveres de 10 zopilotes negros recibidos en un centro de triaje de animales silvestres en Belo Horizonte, Minas Gerais, Brasil. En otras especies de catártidos se han reportado prevalencias bajas para estos endoparásitos, como en el estudio de Rotella et al. (2006) en Cuba, en el que 7.5\% de los zopilotes cabeza roja (Cathartes aura) a los que se les realizó necropsia presentaron helmintos que se identificaron como semejantes a nematodos del género Tetrameres. En otro estudio, Bustamante (2013) analizó muestras fecales de ocho cóndores andinos (Vultur gryphus) (cuatro de vida libre y cuatro en cautiverio) en Perú y detectó huevos de Porrocaecum sp. en una muestra (12.5\%) de un cóndor en cautiverio. Los diferentes resultados obtenidos en el presente estudio y en los estudios anteriormente mencionados pueden deberse a diversos factores como la especie evaluada, el tamaño de la muestra, los métodos utilizados para la detección de helmintos, la época del año en la que se realizó cada estudio, el hábitat de los animales evaluados y su grado de infestación.

Según los resultados del análisis fecal realizado, la fauna helmintológica gastrointestinal de la población de zopilotes negros sujeta a estudio estuvo predominada por un trematodo de la superfamilia Diplostomoidea. Es posible que el grado de infestación con este trematodo en estas aves fuese alto ya que el hallazgo de los huevos se realizó mediante el método directo, con el cual sólo una pequeña cantidad de la muestra a evaluar es utilizada, por lo que un resultado positivo puede ser indicativo de una infestación considerable (Thienpont et al. 2003). Anteriormente han sido encontradas especies pertenecientes a esta superfamilia en $C$. atratus principalmente en Brasil, donde existen registros de Apatemon (Australapatemon) bdellocystis, Strigea vaginata, Strigea falconis brasiliana y una especie de 
Strigea no identificada (Noronha et al. 2009), además del hallazgo en dos muestras fecales de estas aves de huevos de un trematodo de la familia Strigeidae (Lie \& Basch 1967). En Argentina y en Venezuela también se registró la especie Strigea vaginata (Caballero \& Díaz-Ungría, citados en Lunaschi \& Drago 2009; Drago \& Lunaschi 2011). En la presente investigación se observaron además huevos de Echinostomatidae. Dos especies de esta familia se han reportado en zopilotes negros: Paryphostomum segregatum en Brasil, Argentina, Paraguay y Venezuela (Lie \& Basch 1967; Kostadinova et al. 2002; Noronha et al. 2009; Drago \& Lunaschi 2011; Caballero \& Díaz-Ungría, citados en Alves-Pinto 2013) y Petasiger sp. en Argentina (Drago \& Lunaschi 2011).

La mayoría de las especies de trematodos de la superfamilia Diplostomoidea poseen ciclos biológicos en los que participan tres hospederos, pero algunas especies utilizan hasta cuatro (Blasco-Costa \& Locke 2017; López-Hernández 2018). Como primer hospedero intermediario necesitan un gasterópodo y como segundo hospedero intermediario muchas especies utilizan peces, pero otras requieren de anfibios o invertebrados como anélidos o moluscos (Blasco-Costa \& Locke 2017; López-Hernández 2018). Algunas especies involucran en su ciclo biológico a un tercer hospedero intermediario que puede ser paraténico u obligatorio, como ocurre con especies del género Strigea que necesitan de anfibios, reptiles, aves o mamíferos como terceros hospederos intermediarios obligatorios (Blasco-Costa \& Locke 2017). Los hospederos definitivos de los miembros de esta superfamilia son aves, mamíferos y reptiles (Blasco-Costa \& Locke 2017; López-Hernández 2018). Por otro lado, los trematodos de la familia Echinostomatidae tienen ciclos biológicos que involucran tres hospederos (Taylor et al. 2016). El primer hospedero intermediario es un gasterópodo y el segundo hospedero intermediario puede ser un molusco, un anfibio o un pez (Gibson 1996; Tkach et al. 2016). Los hospederos definitivos son aves, mamíferos y reptiles (Gibson 1996; Tkach et al. 2016).

En la presente investigación, aunque se desconoce a qué especies de trematodos pertenecen los huevos encontrados, podrían ser especies que utilizan peces como hospederos intermediarios. Por consiguiente, se considera que una de las fuentes de infestación para los zopilotes negros pudo ser los restos de pescados a los cuales tenían acceso, ya que son utilizados para alimentar a aves piscívoras que se encuentran dentro de la Fundación Protectora de Animales en Vías de Extinción (FAE). Es importante señalar que este pescado, proveniente de un lago cercano al área donde se ubica la FAE, se mantenía en congelación y se descongelaba antes de llevarlo a los recintos de aves piscívoras, pero es probable que, a pesar de esto, algunas metacercarias, es decir las fases infectivas, permanecieran aún viables si la temperatura o el tiempo de congelación eran inadecuados o insuficientes para inactivarlas (Adams et al. 1997; Kaenjampa et al. 2017). Se sabe que en áreas donde tienden a acumularse restos de pescado producto de actividades humanas se puede facilitar y aumentar la transmisión de parásitos a animales silvestres que consumen estos restos (Born-Torrijos et al. 2016), lo que podría ayudar a explicar el posible alto grado de infestación con trematodos en los zopilotes negros evaluados; sin embargo, está fuera del alcance de este estudio afirmar que lo anteriormente mencionado haya sido la causa de la trematodiasis en estas aves. Para ello sería necesario llevar a cabo más investigaciones que permitan, entre otros aspectos, conocer las especies de los trematodos adultos que se encuentran en los zopilotes negros, la presencia de metacercarias y la especie a la que pertenecen en el pescado que estos consumen y otras posibles fuentes de infestación.

Por otra parte, es importante indicar que se puede deducir el papel de estas aves como diseminadoras de, principalmente, una especie de trematodo de la superfamilia Diplostomoidea, cuyos huevos se 
Rev. Ciencias Veterinarias, Vol. 37, N² 2, [32-43], E-ISSN: 2215-4507, julio-diciembre, 2019

DOI: https://doi.org/10.15359/rcv.37-2.4

URL: http://www.revistas.una.ac.cr/index.php/veterinaria/index

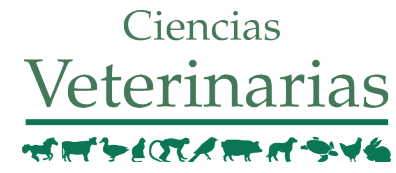

observaron en la mayoría de muestras analizadas. Esto puede contribuir a que otras especies de vida libre susceptibles a la infestación con este trematodo que cohabitan con los zopilotes negros tengan mayor probabilidad de adquirir la trematodiasis, especialmente aves ya que, considerando que su presencia se determinó en una especie de ave (Coragyps atratus), podría no afectar a otros grupos de animales como mamíferos o reptiles. También es importante el hecho de que las especies que pueden actuar como hospederos intermediarios en el ciclo biológico del trematodo, como los peces, suelen verse perjudicadas por los efectos negativos que producen las metacercarias en ellos (Goater et al. 2014; López-Hernández 2018). En cuanto a las aves en cautiverio que se encuentran dentro de la FAE no representa un problema directo el hecho de que los zopilotes estén eliminando estos huevos en las heces, debido a que es muy poco probable que en las condiciones en cautiverio se complete el ciclo de vida de este endoparásito; no obstante, sí podría ser de importancia si el ciclo de vida involucra peces y los zopilotes infestados defecaran en áreas cercanas a donde habitan los peces que luego son utilizados para alimentar a estas aves en cautiverio, lo cual es probable dado que, como se mencionó con anterioridad, este pescado proviene de un lago cercano a la FAE; de esta forma sí se completaría el ciclo. En lo referente a las aves domésticas, como las aves de traspatio que se tienen en viviendas cercanas al área de estudio, no se considera que este trematodo represente un problema ya que los hospederos intermediarios que pueden participar en su ciclo biológico no forman parte de la dieta de estas aves. Finalmente, tampoco se considera que la diseminación de este trematodo en el área represente un riesgo para la salud humana, dado que son muy pocos los miembros de la superfamilia Diplostomoidea que afectan a humanos y sólo se han reportado casos al respecto en Europa y en Asia (Toledo et al. 2014; López-Hernández 2018).

En lo que se refiere a nematodos gastrointestinales, de los tres taxones identificados en el presente estudio, solamente Heterakis sp. ha sido reportado anteriormente en C. atratus; el hallazgo se obtuvo en una muestra fecal recolectada en un recinto de zopilotes negros en un zoológico de Brasil (Frasson et al. 2016). Las otras especies de nematodos que se conoce que han sido encontradas en C. atratus en el tracto gastrointestinal son Tetrameres paradoxus y Tetrameres sp. también en Brasil (Vicente et al. 1995). Estos helmintos no fueron detectados en las muestras fecales analizadas en la presente investigación. Por otro lado, en una de estas muestras se encontró un huevo de un nematodo de la familia Ascarididae que se considera que podría ser del género Porrocaecum; en Perú existen registros de Porrocaecum sp. en otro catártido, el cóndor andino (Sarmiento et al. 1999; Bustamante 2013). En cuanto a nematodos de la familia Trichostrongylidae no se tiene conocimiento de que hayan sido observados con anterioridad en ningún catártido. Por otra parte, con respecto a los acantocéfalos, en C. atratus solamente se ha reportado la especie Oligacanthorhynchus spira en Brasil (Travassos 1917). La especie de acantocéfalo a la que pertenecen los huevos observados en el presente estudio no pudo ser identificada.

Se debe mencionar que es probable que algunos de los huevos detectados en las muestras analizadas, especialmente algunos de los que se encontraron solamente en una de estas muestras, estuvieran presentes en las heces de los zopilotes negros como resultado de que estos hayan consumido animales infestados con helmintos o sus heces, es decir que podrían tratarse de parásitos espurios (Zajac \& Conboy 2012), lo cual se debe tener en cuenta considerando los hábitos alimenticios de estas aves. Por lo anterior, no se puede afirmar que todos los taxones identificados correspondan a parásitos verdaderos de los zopilotes negros muestreados. 
De los dos métodos diagnósticos utilizados en este estudio solamente el método directo es útil para detectar huevos de trematodos y, debido a que estos fueron los huevos con mayor presencia en las muestras analizadas, este fue el método con el que mayores resultados positivos se obtuvieron. Por otro lado, el método de flotación con una solución sobresaturada de azúcar se utiliza para el diagnóstico de huevos menos pesados como los de nematodos y de cestodos y para algunos quistes de protozoos (Zajac \& Conboy 2012; Bowman 2014). Los huevos de trematodos y los de acantocéfalos, al ser más pesados, no flotan en esta solución y, por consiguiente, no son detectados, por lo que son necesarios otros métodos diagnósticos como las técnicas de sedimentación (Zajac \& Conboy 2012; Bowman 2014). En general se recomienda que sea utilizado más de un método coproparasitológico cuando se analizan muestras fecales en busca de helmintos gastrointestinales (Smith 1993; Navone et al. 2005).

Los hallazgos de la investigación aquí presentada constituyen un primer acercamiento al conocimiento de la fauna helmintológica gastrointestinal de Coragyps atratus en Guatemala y pueden aportar información relevante principalmente para el manejo, la medicina y la conservación tanto de esta especie como de otras de vida libre o en cautiverio que habitan la misma área y pueden verse afectadas por la eliminación de los huevos de helmintos en las heces de estos zopilotes. Futuras investigaciones son necesarias para generar más información sobre este campo, tanto en este país como en otros países de la región.

\section{Conclusión}

De acuerdo con lo detectado a través del análisis coproparasitológico realizado, los zopilotes negros evaluados presentaron helmintos gastrointestinales, predominando la infestación con trematodos de una especie de la superfamilia Diplostomoidea.

\section{Agradecimientos}

A la Fundación Protectora de Animales en Vías de Extinción (FAE), en especial a la M.V. Josselyn Esquité, por permitirme realizar el estudio en este lugar y por su valiosa ayuda y hospitalidad durante la realización del mismo.

\section{Referencias}

Adams, A. M., Murrell, K. D. \& Cross, J. H. 1997. Parasites of fish and risks to public health. Rev. Sci. Tech. OIE 16(2): 652-660. DOI: dx.doi.org/10.20506/rst.16.2.1059

Alves-Pinto, H. 2013. Biología e taxonomía de trematódeos transmitidos por moluscos dulciaquícolas na Represa da Pampulha, Belo Horizonte, Minas Gerais, Brasil. Tesis de Doctorado en Parasitología. Universidade Federal de Minas Gerais, Belo Horizonte, Brasil.

Andery, D. de A., Ferreira-Junior, F.C., Araújo, A. V., Vilela, D. A. da R., Marques, M.V.R., Marin, S.Y., Horta, R.S., Ortiz, M.C., Resende, J.S. \& Martins, N.R. da S. 2013. Health assessment of raptors in triage in Belo Horizonte, MG, Brazil. Braz. J. Poult. Sci. 15(3): 247-256. DOI: dx.doi.org/10.1590/ S1516-635X2013000300012 
Rev. Ciencias Veterinarias, Vol. 37, N² 2, [32-43], E-ISSN: 2215-4507, julio-diciembre, 2019

DOI: https://doi.org/10.15359/rcv.37-2.4

Ciencias

URL: http://www.revistas.una.ac.cr/index.php/veterinaria/index

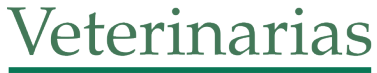

Avery, M. L. \& Lowney, M. 2016. Vultures. Wildlife Damage Management Technical Series. USDA, APHIS, WS National Wildlife Research Center, Fort Collins, Colorado. p. 9-12.

Ballejo, F. \& De Santis, L.J.M. 2013. Dieta estacional del jote cabeza negra (Coragyps atratus) en un área rural y una urbana en el noroeste patagónico. El Hornero 28(1): 7-14.

Basch P.F., DiConza J.J. \& Johnson B.E. 1973. Strigeid trematodes (Cotylurus lutzi) cultured in vitro: Production of normal eggs with continuance of life cycle. J. Parasitol. 59(2): 319-322. DOI: $10.2307 / 3278824$

Bildstein, K. L., Bechard, M. J., Porras, P., Campo, E. \& Farmer, C. J. 2007. Seasonal abundances and distributions of black vultures (Coragyps atratus) and turkey vultures (Cathartes aura) in Costa Rica and Panama: Evidence for reciprocal migration in the neotropics. In Bildstein, K. L., Barber, D. R. \& Zimmerman, A. (Eds.), Neotropical Raptors: Proceedings of the Second Neotropical Raptors Network Congress. Hawk Mountain Sanctuary, Orwigsburg, Pennsylvania. p.47-60.

Blasco-Costa, I. \& Locke, S. A. 2017. Life history, systematics and evolution of the Diplostomoidea Poirier, 1886: Progress, promises and challenges emerging from molecular studies. Adv. Parasitol. 98:167-225. DOI: doi.org/10.1016/bs.apar.2017.05.001

Borgsteede, F.H.M. 1996. The effect of parasites on wildlife. Veterinary Quarterly 18(3): 138-140. DOI: doi.org/10.1080/01652176.1996.9694717

Born-Torrijos, A., Poulin, R., Pérez-del-Olmo, A., Culurgioni, J., Raga, J. A. \& Holzer, A. S. 2016. An optimised multi-host trematode life cycle: fishery discards enhance trophic parasite transmission to scavenging birds. Int. J. Parasitol. 46(11): 745-753. DOI: doi.org/10.1016/j.ijpara.2016.06.005

Bowman, D. 2014. Georgis' Parasitology for Veterinarians, $10^{\text {th }}$ edition. Elsevier Saunders, St. Louis, Missouri. p. 326-398.

Bustamante, J. 2013. Morfometría corporal y parásitos gastrointestinales del cóndor andino (Vultur gryphus) en estado silvestre y cautiverio en los departamentos de Apurímac y Cusco, 2012. Tesis para optar al título de Médico Veterinario y Zootecnista. Universidad Católica de Santa María, Arequipa, Perú.

Chai, J.Y., Sohn, W.M., Chung, H.L., Hong, S.T. \& Lee S.H. 1990. Metacercariae of Pharyngostomum cordatum found from the European grass snake, Rhabdophis tigrina, and its experimental infection to cats. Korean J. Parasitol. 28(3):175-181. DOI: dx.doi.org/10.3347/kjp.1990.28.3.175

De la Cruz, J. R. 1982. Clasificación de zonas de vida de Guatemala a nivel de reconocimiento. Ministerio de Agricultura, Ganadería y Alimentación (MAGA), Guatemala. p.11-36.

Drago, F. B. \& Lunaschi, L. I. 2011. Digenean parasites of Ciconiiform birds from Argentina. Rev. Mex. Biodivers. 82(1): 77-83.

Ferguson-Lees, J. \& Christie, D. A. 2001. Raptors of the World. Houghton Mifflin Harcourt, Boston. p.305-306.

Figueroa-Hernández, L. \& Rodríguez-Zea, M. 2007. Manual de técnicas diagnósticas en parasitología veterinaria. Facultad de Medicina Veterinaria y Zootecnia, Universidad de San Carlos de Guatemala, Guatemala. p.11-14. 
Frasson, L., Wolkmer, P., Soares, J., Rosa, L., Marchesan, C., Ferreira, E., Salomão, E. \& Buiz, T. 2016. Pesquisa de ectoparasitas e analise coproparasitológica de animais silvestres mantidos em cativeiro no Zoológico de Cachoeira do Sul-RS do Brasil. I Conferência e Mostra Científica Internacional Em Bem-Estar Animal. Itapiranga, Santa Catarina, Brasil.

Gibson, D. I. 1996. Trematoda. In Margolis, L. \& Kabata, Z. (Eds.), Guide to the parasites of fishes of Canada. Part IV. Canadian Special Publication of Fisheries and Aquatic Sciences 124, Ottawa. p.133.

Globokar, M., Fischer, D. \& Pantchev, N. 2017. Occurrence of endoparasites in captive birds between 2005 to 2011 as determined by faecal flotation and review of literature. Berliner und Münchener tierärztliche Wochenschrift 130(11/12): 461-473. DOI: 10.2376/0005-9366-16094

Goater, T.M., Goater, C.P. \& Esch, G.W. 2014. Parasitism: The Diversity and Ecology of Animal Parasites, $2^{\text {nd }}$ Edition. Cambridge University Press, New York, p.396-431.

Hong S.T., Cho T.K., Hong S.J., Chai J.Y., Lee S.H. \& Seo B.S. 1984. Fifteen human cases of Fibricola seoulensis infection in Korea. Korean J. Parasitol. 22(1): 61-65. DOI: dx.doi.org/10.3347/ kjp.1984.22.1.61

Houston, D. \& Kirwan, G. M. 2019. American Black Vulture (Coragyps atratus). https://www.hbw.com/ species/american-black-vulture-coragyps-atratus. (Accessed 4 March 2019).

INSIVUMEH (Instituto Nacional de Sismología, Vulcanología, Meteorología e Hidrología). 2018. Normales climáticas. http://www.insivumeh.gob.gt/normas-climaticas/ (Accessed 11 November 2018).

Kaenjampa, P., Tangkawattana, S., Smith, J., Sukon, P. \& Tangkawattana, P. 2017. Elimination of Haplorchis taichui metacercaria in cyprinoid fish with freezing temperature and soured fish (plasom) with salinity. Southeast Asian J. Trop. Med. Public Health 48(4): 777-785.

Kostadinova, A., Vaucher, C. \& Gibson, D. I. 2002. Redescriptions of two echinostomes from birds in Paraguay, with comments on Drepanocephalus Dietz, 1909 and Paryphostomum Dietz, 1909 (Digenea: Echinostomatidae). Systematic Parasitology 53(2): 147-158. DOI: doi. org/10.1023/A:1020418124561

Krone, O. 2007. Endoparasites. In: Bird D.M. \& Bildstein K.L. (Eds.), Raptor research and management techniques. Hancock House, Surrey, British Columbia and Blaine, Washington. p. 318-328.

Lie, K. J. \& Basch, P. F. 1967. The life history of Paryphostomum segregatum Dietz, 1909. J. Parasitol. 53(2): 280-286. DOI: $10.2307 / 3276576$

Loker, E.S. \& Hofkin, B.V. 2015. Parasitology: A conceptual approach. Garland Science, Taylor \& Francis Group, New York and Abingdon, Oxfordshire. p. 219-221, 344-345.

López-Hernández, D. D. 2018. Estudo morfológico e molecular de larvas de Diplostomoidea (Trematoda: Digenea) transmitidas por moluscos dulciaquícolas em Belo Horizonte, Minas Gerais, Brasil. Tesis de Maestría en Parasitología. Universidade Federal de Minas Gerais, Belo Horizonte, Brasil.

Lunaschi, L. I. \& Drago, F. B. 2009. Species of Strigea (Digenea: Strigeidae), parasites of the savanna hawk Buteogallus meridionalis (Aves: Accipitridae) from Argentina, with the description of a new species. Folia Parasitol. 56(4): 268-274. DOI: 10.14411/fp.2009.031 
Rev. Ciencias Veterinarias, Vol. 37, N² 2, [32-43], E-ISSN: 2215-4507, julio-diciembre, 2019

DOI: https://doi.org/10.15359/rcv.37-2.4

Ciencias

URL: http://www.revistas.una.ac.cr/index.php/veterinaria/index

Veterinarias

Navone, G.T., Gamboa, M.I., Kozubsky, L.E., Costas, M.E., Cardozo, M.S., Sisliauskas, M.N. \& González, M. 2005. Estudio comparativo de recuperación de formas parasitarias por tres diferentes métodos de enriquecimiento coproparasitológico. Parasitol. latinoam. 60(3-4):178-181. DOI: dx.doi. org/10.4067/S0717-77122005000200014

Noronha, D., Sá, M. R., Knoff, M., Muniz-Pereira, L. C. \& Pinto, R. M. 2009. Adolpho Lutz e a coleção helmintológica do Instituto Oswaldo Cruz. Museu Nacional, Rio de Janeiro. p.73-136.

Raffaele, H., Wiley, J., Garrido, O., Keith, A. \& Raffaele, J. 2003. Birds of the West Indies. Princeton University Press, Princeton, New Jersey. p.80.

Rotella, I. J., Silveira, E. A., Delgado, L., González, O., Remón, S., Rojas, D., Gónzalez, J.A., Manso, M.J., Perdomo, J.R. \& Campos, R. 2006. Contribución al conocimiento de la epizootiología y biología del Cathartes aura Lin. REDVET 7(1): 1-12.

Sarmiento, L., Tantaleán, M. \& Huiza, A. 1999. Nematodos parásitos del hombre y de los animales en el Perú. Rev. Peru. Parasitol. 14(1-2): 9-65.

Schlatter, R., Reinhardt, G. \& Burchard, L. 1978. Estudio del jote (Coragyps atratus foetens, Lichtenstein) en Valdivia: Etología carroñera y rol en diseminación de agentes patógenos. Arch. Med. Vet. 10(2): 111-127.

Smith, S.A. 1993. Diagnosis and treatment of helminths in birds of prey. In: Redig, P.T., Cooper, J.E., Remple, D., \& Hunter, B. (Eds.), Raptor Biomedicine. University of Minnesota Press, Minneapolis, Minnesota. p. 21-27.

Taylor, M.A., Coop, R.L. \& Wall, R.L. 2016. Veterinary Parasitology, $4^{\text {th }}$ edition. Wiley Blackwell, Ames, Iowa. p. 82, 261-267.

Thienpont, D., Rochette, F. \& Vanparijs, O.F.J. 2003. Diagnosing helminthiasis by coprological examination. Janssen Animal Health, Beerse, Belgium. p.29-203.

Tkach, V. V., Kudlai, O. \& Kostadinova, A. 2016. Molecular phylogeny and systematics of the Echinostomatoidea Looss, 1899 (Platyhelminthes: Digenea). Int. J. Parasitol. 46(3): 171-185. DOI: doi.org/10.1016/j.ijpara.2015.11.001

Toledo, R., Muñoz-Antoli, C. \& Esteban, J.G. 2014. Intestinal Trematode Infections. In: Toledo, R. \& Fried, B. (Eds.), Digenetic tremadode infections. Advances in Experimental Medicine and Biology. Springer, New York. p. 201-240. DOI: doi.org/10.1007/978-1-4939-0915-5_7

Travassos, L. 1917. Contribuições para o conhecimento da fauna helmintolojica brazileira. VI. Revisão dos acantocefalos brazileiros. Parte I. Fam. Gygantorhynchidae Hamann, 1892. Mem. Inst. Oswaldo Cruz 9(1): 5-62. DOI: dx.doi.org/10.1590/S0074-02761917000100001

Vicente, J. J., Rodrigues, H. de O., Gomes, D. C. \& Pinto, R. M. 1995. Nematóides do Brasil. Parte IV: Nematóides de aves. Rev. Bras. Zool. 12: 1-273. DOI: dx.doi.org/10.1590/S0101-81751995000500001

Zajac, A. M. \& Conboy, G. A. 2012. Veterinary clinical parasitology, 8th ed. Wiley-Blackwell, Ames, Iowa. p.4-14, 19-169. 